\title{
Modulation Spaces, BMO, and the Balian-Low Theorem
}

\author{
Ramazan Tinaztepe \\ Department of Mathematics Education, Zirve University, \\ 27260, Gaziantep, Turkey \\ ramazan.tinaztepe@zirve.edu.tr \\ Christopher Heil \\ School of Mathematics, Georgia Institute of Technology, \\ Atlanta, GA 30332-0160 USA \\ heil@math.gatech.edu
}

\begin{abstract}
The modulation spaces $M_{m}^{p, q}\left(\mathbb{R}^{d}\right)$ quantify the time-frequency concentration of functions and distributions. The first main result of this paper proves embeddings of certain modulation spaces into VMO, the space of functions with vanishing mean oscillation. The second main result proves that the Zak transform maps certain modulation spaces on $\mathbb{R}^{d}$ into modulation spaces on $\mathbb{R}^{2 d}$. These two results allow us to give a Balian-Low-type of uncertainty principle for Gabor systems in the setting of modulation spaces.
\end{abstract}

Key words and phrases : Balian-Low Theorem, BMO, frames, Gabor systems, modulation spaces, time-frequency analysis, Poincare Inequality, Short-time Fourier transform, VMO, Zak transform

2000 AMS Mathematics Subject Classification - Primary 42C15, 42C25; Secondary 46C15

\section{Introduction}

Time-frequency analysis interprets the short-time Fourier transform as a measure of simultaneous time and frequency information. To achieve a quantitative analysis, Feichtinger introduced a class of Banach spaces, called the modulation spaces, which measure concentration in terms of a weighted mixed norm on the short-time Fourier transform [8], [11].

The modulation spaces are the "right" spaces for time-frequency analysis and they occur in many problems in the same way that Besov spaces are attached to wavelet theory and issues of smoothness. Many classical spaces are welldescribed by wavelets, but recently the modulation spaces have been successfully 
used to address problems not suited to traditional techniques, e.g., the analysis of pseudodifferential operators with nonsmooth symbols [4], [13], or the modeling of narrowband wireless communication channels [18], [19], [20].

For $p, q \neq 2$, the modulation spaces $M_{m}^{p, q}\left(\mathbb{R}^{d}\right)$ do not coincide with any of the Besov spaces $B_{s}^{p, q}\left(\mathbb{R}^{d}\right)$ or the Triebel-Lizorkin spaces $F_{s}^{p, q}\left(\mathbb{R}^{d}\right)$. The question of which of these classical function spaces embed into the modulation spaces or vice versa is a natural one. The Sobolev embedding theorem $H^{s}\left(\mathbb{R}^{d}\right) \subseteq C^{k}\left(\mathbb{R}^{d}\right)$ for $s>k+\frac{1}{2} d$ is an example of such a result, as $H^{s}\left(\mathbb{R}^{d}\right)$ is a modulation space (with $p=2$ ) and $C^{k}\left(\mathbb{R}^{d}\right)$ is a Besov space. The first systematic study of embeddings of Besov spaces into modulation spaces was by Okoudjou [16], with further results by Toft [21], [22].

One of the important results in time-frequency analysis that is stated in the setting of weighted Sobolev spaces is the Balian-Low theorem. In this paper, we investigate how modulation spaces relate to the Balian-Low theorem, and state a Balian-Low-type theorem for Gabor frames in the setting of modulation spaces. Gautam's results [10] make clear that membership in VMO (the space of functions of vanishing mean oscillation) is central to the Balian-Low Theorem. The embedding of the modulation spaces into BMO or VMO is an important issue in establishing the Balian-Low theorem in terms of modulation spaces.

The first of our main results proves embeddings of certain modulation spaces into VMO. While there are many known embeddings of classical function spaces into $\mathrm{BMO}$ or $\mathrm{VMO}$, the question for the modulation spaces has not previously been addressed.

Another important time-frequency tool which plays a key role in the BalianLow theorem is the Zak transform, also known as Weil-Brezin map (first introduced by Gelfand [14]). For a function $f \in L^{2}\left(\mathbb{R}^{d}\right)$, its Zak transform $Z f$ is defined by

$$
Z f(x, \omega)=\sum_{k \in \mathbb{Z}^{d}} f(x-k) e^{2 \pi i k \cdot \omega}
$$

where the series converges unconditionally in $L^{2}\left(\mathbb{R}^{d}\right)[11] . Z f$ provides a useful time-frequency representation of $f$. The Zak transform's importance in the Balian-Low theorem comes from the fact that the properties of the Zak transform of $f$ can be explicitly tied to whether the associated Gabor system is a frame or not.

In [10], Gautam gives a time-frequency characterization of the Zak transform of a function having certain smoothness and decay properties, quantified in terms of membership in an inhomogeneous Sobolev space (see Lemma 2.3 in [10]). Since the time-frequency content of a function is best quantified by the modulation spaces, this motivated our investigation of the modulation space characterization of $Z f$. Our second main result proves that the Zak transform maps certain modulation spaces on $\mathbb{R}^{d}$ into modulation spaces on $\mathbb{R}^{2 d}$. This provides a generalization of Gautam's result, and in fact we obtain his embedding 
as a corollary. In proving this result, we appeal to Gabor analysis rather than the tools of classical analysis employed in [10]. In particular, we use Janssen's characterization of modulation spaces via the Zak transform [15], which provides a practical approach to our problem and allows us to extend the result to higher dimensions.

Finally, combined with our BMO embeddings, we see that Zak transform maps certain modulation spaces into VMO. As a consequence of these facts, we obtain a Balian-Low-type theorem for modulation spaces that is equivalent to the classical statement of the Balian-Low Theorem.

Our paper is organized as follows. In Section 2 we give some preliminaries on the modulation spaces and the Zak transform. Section 3 contains our results on embeddings of modulation spaces into BMO, and Section 4 gives a modulation space characterization of the Zak transform. Finally, Section 5 discusses the connection to the Balian-Low theorem.

\section{Preliminaries: Modulation Spaces and the Zak Transform}

\subsection{General Notation}

$\mathcal{S}\left(\mathbb{R}^{d}\right)$ will denote the class of Schwartz functions on $\mathbb{R}^{d}$, and $\mathcal{S}^{\prime}\left(\mathbb{R}^{d}\right)$ is its topological dual, the space of tempered distributions. We use a Fourier transform on $L^{1}\left(\mathbb{R}^{d}\right)$ normalized as

$$
\mathcal{F} f(\omega)=\widehat{f}(\omega)=\int_{\mathbb{R}^{d}} f(x) e^{-2 \pi i \omega \cdot x} d x .
$$

This extends to a unitary operator on $L^{2}\left(\mathbb{R}^{d}\right)$, and by duality to an operator mapping $\mathcal{S}^{\prime}\left(\mathbb{R}^{d}\right)$ into itself.

The Fourier coefficients of $F \in L^{1}[0,1)^{d}$ are

$$
\widehat{F}(n)=\int_{[0,1)^{d}} F(x) e^{-2 \pi i n \cdot x} d x, \quad n \in \mathbb{Z}^{d} .
$$

To avoid dealing with too many intermediate constants, we will write $A \lesssim B$ to mean that $A(x) \leq C B(x)$ for all $x$ where $C$ is a constant independent of $x$ (and possibly other parameters).

Given $x \in \mathbb{R}^{d}$, we let $x^{(r)}$ denote the $r^{\text {th }}$ component of $x$. Also $|x|$ will denote the Euclidean norm when $x \in \mathbb{R}^{d}$ and absolute value when $x$ is a real or complex number.

\subsection{The Modulation Spaces}

We introduce the modulation spaces and give some of their properties that are relevant to the results of this paper, referring to Gröchenig's text [11] for full 
details.

The Short-Time Fourier Transform (STFT) of a function $f$ with respect to a window function $g$ is

$$
V_{g} f(x, \omega)=\int_{\mathbb{R}^{d}} f(t) \overline{g(t-x)} e^{-2 \pi i t \cdot \omega} d t, \quad(x, \omega) \in \mathbb{R}^{2 d},
$$

whenever this is defined. If $f, g$ lie in dual spaces (typically $f \in \mathcal{S}^{\prime}\left(\mathbb{R}^{d}\right)$ and $g \in \mathcal{S}\left(\mathbb{R}^{d}\right)$, or $f, g \in L^{2}\left(\mathbb{R}^{d}\right)$ ), then $V_{g} f$ is uniformly continuous on $\mathbb{R}^{2 d}$, and the following fundamental identity of the time-frequency analysis is satisfied:

$$
V_{g} f(x, \omega)=e^{-2 \pi i x \cdot \omega} V_{\widehat{g}} \widehat{f}(\omega,-x) .
$$

A weight function is a positive function on $\mathbb{R}^{n}$. We say that a positive function $v$ on $\mathbb{R}^{2 d}$ is submultiplicative if $v\left(z_{1}+z_{2}\right) \leq v\left(z_{1}\right) v\left(z_{2}\right)$ for all $z_{1}, z_{2} \in \mathbb{R}^{2 d}$. If $v$ is submultiplicative, then a positive function $m$ on $\mathbb{R}^{2 d}$ is $v$-moderate if there exists a constant $C>0$ such that $m\left(z_{1}+z_{2}\right) \leq C v\left(z_{1}\right) m\left(z_{2}\right)$ for all $z_{1}$, $z_{2} \in \mathbb{R}^{2 d}$. Two weights $m_{1}, m_{2}$ are equivalent if there exists a constant $C>0$ such that $C^{-1} m_{1}(z) \leq m_{2}(z) \leq C m_{1}(z)$ for all $z \in \mathbb{R}^{2 d}$. The standard class of weights on $\mathbb{R}^{2 d}$ are weights of polynomial type $v_{s}(z)=(1+|z|)^{s}, s \in \mathbb{R}$, where $z=(x, w) \in \mathbb{R}^{2 d}$ as usual and $s \geq 0$. If $s \geq 0$ then $v_{s}$ is submultiplicative, while if $s \leq 0$ then $v_{s}$ is $v_{-s}$-moderate. The weights

$$
m_{s}(z)=\left(1+|z|^{2}\right)^{s / 2}, \quad s \in \mathbb{R},
$$

are typical and are used, for example, to define the Bessel potential spaces. The Bessel potential spaces are defined as

$$
H^{s}\left(\mathbb{R}^{d}\right)=\left\{f \in \mathcal{S}^{\prime}\left(\mathbb{R}^{d}\right):\left(\int_{\mathbb{R}^{d}}|\hat{f}(w)|^{2}\left|m_{s}(w)\right|^{2} d w\right)^{\frac{1}{2}}<\infty\right\} .
$$

We remark explicitly that $v_{s}(z)$ is equivalent to the weight $m_{s}(z)$.

Let $m$ be a weight function on $\mathbb{R}^{2 d}$ and fix $1 \leq p, q<\infty$. The weighted mixed-norm space $L_{m}^{p, q}\left(\mathbb{R}^{2 d}\right)$ consists of all (Lebesgue) measurable functions on $\mathbb{R}^{2 d}$ such that the norm

$$
\|F\|_{L_{m}^{p, q}\left(\mathbb{R}^{2 d}\right)}=\left(\int_{\mathbb{R}^{d}}\left(\int_{\mathbb{R}^{d}}|F(x, \omega)|^{p} m(x, \omega)^{p} d x\right)^{q / p} d \omega\right)^{1 / q}
$$

is finite, with the usual adjustments if $p=\infty$ or $q=\infty$. If $m=m_{s}$ then we write $L_{s}^{p}=L_{m_{s}}^{p}$. If $p=q$ then we write $L_{m}^{p}$ for $L_{m}^{p, p}$.

Fix a nonzero window function $g \in \mathcal{S}\left(\mathbb{R}^{d}\right)$, a $v$-moderate weight function $m$ on $\mathbb{R}^{2 d}$, and $1 \leq p, q \leq \infty$. Then the modulation space $M_{m}^{p, q}\left(\mathbb{R}^{d}\right)$ consists of all tempered distributions $f \in \mathcal{S}^{\prime}\left(\mathbb{R}^{d}\right)$ such that $V_{g} f \in L_{m}^{p, q}\left(\mathbb{R}^{2 d}\right)$. The norm on $M_{m}^{p, q}\left(\mathbb{R}^{d}\right)$ is

$$
\|f\|_{M_{m}^{p, q}\left(\mathbb{R}^{d}\right)}=\left\|V_{g} f\right\|_{L_{m}^{p, q}\left(\mathbb{R}^{2 d}\right)} .
$$


If $p=q$ then we write $M_{m}^{p}\left(\mathbb{R}^{d}\right)$ instead of $M_{m}^{p, p}\left(\mathbb{R}^{d}\right)$. This definition of $M_{m}^{p, q}\left(\mathbb{R}^{d}\right)$ is independent of the choice of window function $g \in \mathcal{S}\left(\mathbb{R}^{d}\right)$ in the sense of equivalent norms. Further, $M_{m}^{p, q}\left(\mathbb{R}^{d}\right)$ is a Banach space, and $\mathcal{S}\left(\mathbb{R}^{d}\right)$ is a subspace of $M_{m}^{p, q}\left(\mathbb{R}^{d}\right)$ if $|m(z)| \lesssim(1+|z|)^{N}$ for some $N$. Since $\left|V_{g} f(x, \omega)\right|=\left|V_{\widehat{g}} \widehat{f}(\omega,-x)\right|$, we have $\|\widehat{f}\|_{M_{\tilde{m}}^{p}}=\|f\|_{M_{m}^{p}}$ where $\widetilde{m}(x, \omega)=m(-\omega, x)$.

The following proposition identifies some of the modulation spaces with wellknown function spaces, see [11, Prop. 11.3.1].

Proposition 2.1. Fix $g \in \mathcal{S}\left(\mathbb{R}^{d}\right) \backslash\{0\}$.

(a) If $|f(x)| \lesssim(1+|x|)^{-s}$ and $s>d$, then $\left|V_{g} f(x, \omega)\right| \lesssim(1+|x|)^{-s}$. If $|\widehat{f}(\omega)| \lesssim$ $(1+|\omega|)^{-s}$ and $s \geq d$, then $\left|V_{g} f(x, \omega)\right| \lesssim(1+|\omega|)^{-s .}$

(b) If $m(x, \omega)=m(x)$, then $M_{m}^{2}\left(\mathbb{R}^{d}\right)=L_{m}^{2}\left(\mathbb{R}^{d}\right)$, the weighted $L^{2}$ space.

(c) If $m(x, \omega)=m(\omega)$, then $M_{m}^{2}\left(\mathbb{R}^{d}\right)=\mathcal{F} L_{m}^{2}\left(\mathbb{R}^{d}\right)$. In particular, by taking $m_{s}(\omega)=\left(1+|\omega|^{2}\right)^{s / 2}$ where $s \in \mathbb{R}$, the space $M_{m_{s}}^{2}\left(\mathbb{R}^{d}\right)$ coincides with the Bessel potential (or Sobolev) space $H^{s}\left(\mathbb{R}^{d}\right)$.

For a choice of appropriate weights we can identify the space of functions with certain smoothness and decay properties as a modulation space. In light of Proposition 2.1, the following fact can be easily proved.

Proposition 2.2. Fix $s_{1}, s_{2}>0$.

(a) Assume $1+m_{s_{1}}(\omega)^{2}+m_{s_{2}}(x)^{2} \gtrsim m(x, \omega)^{2}$. If $f \in H^{s_{1}}\left(\mathbb{R}^{d}\right)$ and $\widehat{f} \in$ $H^{s_{2}}\left(\mathbb{R}^{d}\right)$, then $f \in M_{m}^{2}\left(\mathbb{R}^{d}\right)$.

(b) Assume $m(x, \omega) \gtrsim \max \left\{m_{s_{1}}(\omega), m_{s_{2}}(x)\right\}$. If $f \in M_{m}^{2}\left(\mathbb{R}^{d}\right)$, then $f \in$ $H^{s_{1}}\left(\mathbb{R}^{d}\right)$ and $\widehat{f} \in H^{s_{2}}\left(\mathbb{R}^{d}\right)$.

Corollary 2.3. Fix $s_{1}, s_{2}>0$, and set $m(x, \omega)=\left(1+m_{s_{1}}(\omega)^{2}+m_{s_{2}}(x)^{2}\right)^{1 / 2}$. Then

$$
f \in H^{s_{1}}\left(\mathbb{R}^{d}\right) \cap L_{s_{2}}^{2}\left(\mathbb{R}^{d}\right) \Longleftrightarrow f \in H^{s_{1}}\left(\mathbb{R}^{d}\right), \widehat{f} \in H^{s_{2}}\left(\mathbb{R}^{d}\right) \Longleftrightarrow f \in M_{m}^{2}\left(\mathbb{R}^{d}\right) .
$$

Discrete mixed-norm spaces $\ell_{m}^{p, q}\left(\mathbb{Z}^{2 d}\right)$ are defined as follows. If $m$ is a $v$ moderate weight, then $\ell_{m}^{p, q}\left(\mathbb{Z}^{2 d}\right)$ consists of all sequences $\left(a_{k, n}\right)_{k, n \in \mathbb{Z}^{d}}$ for which the norm

$$
\left\|a_{k, n}\right\|_{\ell_{m}^{p, q}\left(\mathbb{Z}^{2 d}\right)}=\left(\sum_{n \in \mathbb{Z}^{d}}\left(\sum_{k \in \mathbb{Z}^{d}}\left|a_{k, n}\right|^{p} m(k, n)^{p}\right)^{q / p}\right)^{1 / q}
$$

is finite, with the usual adjustments if $p$ or $q$ is infinite. If $p=q$, then we write $\ell_{m}^{p}\left(\mathbb{Z}^{2 d}\right)$.

We will need the following version of Young's Inequality for convolution in weighted sequence spaces (see [11] for proof). 
Proposition 2.4. Let $m$ be v-moderate weight. Given $a=\left(a_{k, n}\right) \in \ell_{v}^{1}\left(\mathbb{Z}^{2 d}\right)$ and $b=\left(b_{k, n}\right) \in \ell_{m}^{p, q}\left(\mathbb{Z}^{2 d}\right)$,

$$
\|a * b\|_{\ell_{m}^{p, q}} \lesssim\|a\|_{\ell_{v}^{1}}\|b\|_{\ell_{m}^{p, q}}
$$

The modulation spaces possess unconditional bases, and the sequence space associated with the basis for $M_{m}^{p, q}\left(\mathbb{R}^{d}\right)$ is the mixed-norm space $\ell_{m}^{p, q}\left(\mathbb{Z}^{2 d}\right)$. As a consequence, the embeddings within the class of modulation spaces follow the embeddings within the class of the sequence spaces.

Theorem 2.5 (Theorem 12.2.2, [11]). If $1 \leq p_{1} \leq p_{2} \leq \infty, 1 \leq q_{1} \leq q_{2} \leq \infty$, and $m^{\prime} \lesssim m$, then $M_{m}^{p_{1}, q_{1}}$ embeds continuously into $M_{m^{\prime}}^{p_{2}, q_{2}}$.

\subsection{The Zak Transform}

The Zak transform of a function $f \in L^{2}\left(\mathbb{R}^{d}\right)$ is the function $Z f$ on $\mathbb{R}^{2 d}$ defined (almost everywhere) by

$$
Z f(x, \omega)=\sum_{k \in \mathbb{Z}^{d}} f(x-k) e^{2 \pi i k \cdot \omega} .
$$

The series defining $Z f$ converges unconditionally in $L^{2}\left([0,1)^{d}\right)$, and the Zak transform is a unitary isomorphism of $L^{2}\left(\mathbb{R}^{d}\right)$ onto $L^{2}\left([0,1)^{d}\right)$. Zf satisfies the following quasiperiodicity relations:

$$
\begin{aligned}
& Z f(x+n, \omega)=e^{2 \pi i \omega \cdot n} Z f(x, \omega), \\
& Z f(x, \omega+n)=Z f(x, \omega) .
\end{aligned}
$$

A consequence of the quasiperiodicity relations is that if $Z f$ is continuous on $\mathbb{R}^{2 d}$, then $Z f$ has a zero in every unit square in $\mathbb{R}^{2 d}$.

The Zak transform maps a function in $\mathcal{S}\left(\mathbb{R}^{d}\right)$ to an infinitely differentiable function on $\mathbb{R}^{2 d}$. A useful property of the Zak Transform is the following equality which describes the Fourier coefficients of $Z f \cdot \overline{Z g}$ as samples of the STFT of $f$ with respect to the window $g$ : Given $f, g \in L^{2}\left(\mathbb{R}^{d}\right)$,

$$
\int_{[0,1)^{d}} \int_{[0,1)^{d}} Z f(x, \omega) \overline{Z g(x, \omega)} e^{-2 \pi i n \cdot x} e^{-2 \pi i m \cdot \omega} d x d \omega=V_{g} f(-m, n) .
$$

This fact allows a characterization of the modulation spaces via the Zak transform. The following result is due to Janssen [15], and we also mention that a different characterization of the modulation spaces was obtained earlier by Walnut [23].

Theorem 2.6. Let $m$ be a v-moderate weight. Let $1 \leq p, q \leq \infty$ and let $g_{r} \in M_{v}^{1}\left(\mathbb{R}^{d}\right)$ for $r=1, \ldots, N$ be such that the functions $Z g_{r}$ have no common 
zeros. If $f \in L^{2}\left(\mathbb{R}^{d}\right)$, then $f \in M_{m}^{p, q}\left(\mathbb{R}^{d}\right)$ if and only if each $Z f \cdot \overline{Z g_{r}}$ has a Fourier series

$$
\sum_{k, \ell} c_{k, \ell}^{(r)} e^{2 \pi i k \cdot x+2 \pi i \ell \cdot \omega}
$$

such that $\left(c_{\ell,-k}\right)_{k, \ell \in \mathbb{Z}^{d}} \in \ell_{m}^{p, q}\left(\mathbb{Z}^{2 d}\right)$.

It is necessary to take $N \geq 2$ in Theorem 2.6 because the functions $Z g_{r}$ will be continuous and therefore will have zeros.

In the sequel, we will use the Zak transform of functions defined on $\mathbb{R}^{d}$ and the Zak transform of functions defined on $\mathbb{R}^{2 d}$. The intended domain will be clear from context.

\section{Embedding of Modulation Spaces into VMO}

We recall that $\mathrm{BMO}\left(\mathbb{R}^{d}\right)$ is the space of functions (modulo constants) that have bounded mean oscillation on $\mathbb{R}^{d}$ :

$$
\operatorname{BMO}\left(\mathbb{R}^{d}\right)=\left\{f \in L_{\mathrm{loc}}^{1}\left(\mathbb{R}^{d}\right):\|f\|_{\mathrm{BMO}}=\sup _{Q}\left(f_{Q}\left|f(x)-f_{Q} f\right| d x\right)<\infty\right\},
$$

where the supremum is taken over all cubes $Q$ in $\mathbb{R}^{d}$, and

$$
f_{E} g=\frac{1}{|E|} \int_{E} g
$$

denotes the average of a function over a Lebesgue measurable set $E$.

The space $\operatorname{VMO}\left(\mathbb{R}^{d}\right)$ of functions having vanishing mean oscillation on $\mathbb{R}^{d}$ consists of those functions $f \in \mathrm{BMO}\left(\mathbb{R}^{d}\right)$ such that

$$
\lim _{a \rightarrow 0} \sup _{|Q| \leq a}\left(f_{Q}\left|f(x)-f_{Q} f\right| d x\right)=0 .
$$

Equivalently, $\operatorname{VMO}\left(\mathbb{R}^{d}\right)$ is the closure of the uniformly continuous functions in BMO-norm. We will work mostly with a local version of VMO; given a compact set $K$ we define $\operatorname{VMO}(K)$ to be the set of functions satisfying the limit condition in equation (3.1) for cubes $Q \subset K$. For details on VMO, we refer to [17].

Now we will obtain an embedding of a certain class of functions defined on $\mathbb{R}^{2}$ into $\mathrm{BMO}\left(\mathbb{R}^{2}\right)$. Although this embedding (Theorem 3.3) follows from the Sobolev embedding theorem in [5] for the case $n=p=2$, for completeness we give a direct proof via Poincare's inequality [7] for functions defined on $\mathbb{R}^{2}$.

The Sobolev space $W^{1, p}(U)$ consists of locally integrable functions $f: U \rightarrow \mathbb{R}$ such that each partial derivative of $f$ exists in the weak sense and belongs to $L^{p}(U)$. 
Theorem 3.1 (Poincare's Inequality). If $1 \leq p \leq \infty$ then

$$
\left\|u-f_{B(x, r)} u\right\|_{L^{p}(B(x, r))} \lesssim r\|D u\|_{L^{p}(B(x, r))}
$$

for each ball $B(x, r) \subseteq \mathbb{R}^{d}$ and each function $u \in W^{1, p}(B(x, r))$.

Corollary 3.2. For all $u \in W^{1,2}\left(\mathbb{R}^{2}\right) \cap L^{1}\left(\mathbb{R}^{2}\right)$ we have

$$
\|u\|_{\mathrm{BMO}\left(\mathbb{R}^{2}\right)} \lesssim\|D u\|_{L^{2}\left(\mathbb{R}^{2}\right)}
$$

Proof. If we take $p=1$ and $d=2$ in Poincare's Inequality and apply Hölder's Inequality, we obtain

$$
\begin{aligned}
f_{B(x, r)}\left|u(y)-f_{B(y, r)} u\right| d y & \lesssim r f_{B(x, r)}|D u| \\
& =\frac{r}{|B(x, r)|} \int_{B(x, r)}|D u| \\
& \leq \frac{r}{|B(x, r)|}|B(x, r)|^{1 / 2}\left(\int_{B(x, r)}|D u|^{2}\right)^{1 / 2} \\
& \lesssim\left(\int_{\mathbb{R}^{2}}|D u|^{2}\right)^{1 / 2} .
\end{aligned}
$$

Now we can prove our first main theorem, on the embedding of weighted modulation spaces into $\mathrm{VMO}\left(\mathbb{R}^{2}\right)$.

Theorem 3.3. Fix $1 \leq p \leq 2$. Assume that:

(a) $v$ is a submultiplicative weight on $\mathbb{R}^{4}$ such that $v(z) \lesssim(1+|z|)^{N_{0}}$ for some $N_{0} \in \mathbb{N}$,

(b) $\widetilde{m}$ is a v-moderate weight on $\mathbb{R}^{4}$ such that $\widetilde{m}(x, \xi) \gtrsim\left(1+|\xi|^{2}\right)^{1 / 2}$.

Then $M_{\widetilde{m}}^{p}\left(\mathbb{R}^{2}\right) \subseteq \operatorname{VMO}\left(\mathbb{R}^{2}\right)$ with

$$
\|f\|_{\mathrm{BMO}} \lesssim\|f\|_{M_{\tilde{m}}^{p}\left(\mathbb{R}^{2}\right)}
$$

Proof. The Schwartz class is dense in $M_{\widetilde{m}}^{p}\left(\mathbb{R}^{2}\right)$, so consider a fixed $f \in \mathcal{S}\left(\mathbb{R}^{2}\right)$. Setting $m(x)=\left(1+|x|^{2}\right)^{1 / 2}$ and applying Corollary 3.2 and the Plancherel Equality, we compute that

$$
\begin{aligned}
\|f\|_{\mathrm{BMO}\left(\mathbb{R}^{2}\right)} & \lesssim\|D f\|_{L^{2}\left(\mathbb{R}^{2}\right)} \\
& \lesssim\||\xi| \widehat{f}(\xi)\|_{2}
\end{aligned}
$$




$$
\begin{aligned}
& \lesssim\left\|\left(1+|\xi|^{2}\right)^{1 / 2} \widehat{f}(\xi)\right\|_{2} \\
& =\|\widehat{f}\|_{L_{m}^{2}} .
\end{aligned}
$$

If we write $m(x, \xi)=m(x)$ then by Proposition 2.1(b) we have

$$
\|\widehat{f}\|_{L_{m}^{2}} \lesssim\|\widehat{f}\|_{M_{m}^{2}} .
$$

Now let $m^{\prime}(x, w)=\widetilde{m}(-w, x)$. By assumption we have $m \lesssim m^{\prime}$, which by Theorem 2.5 implies that

$$
\|\widehat{f}\|_{M_{m}^{2}} \lesssim\|\widehat{f}\|_{M_{m^{\prime}}^{p}}=\|f\|_{M_{\tilde{m}}^{p}} .
$$

An extension by density argument establishes that $\|f\|_{\mathrm{BMO}\left(\mathbb{R}^{2}\right)} \lesssim\|f\|_{M_{\tilde{m}}^{p}}$ for all $f \in M_{\widetilde{m}}^{p}\left(\mathbb{R}^{2}\right)$. Moreover, since VMO is the closure of the uniformly continuous functions in BMO-norm, this also implies that $M_{\widetilde{m}}^{p}\left(\mathbb{R}^{2}\right) \subseteq \operatorname{VMO}\left(\mathbb{R}^{2}\right)$.

\section{Mapping Properties of the Zak Transform on Mod- ulation Spaces}

Our second main theorem describes the mapping properties of the Zak transform acting on the modulation spaces.

Theorem 4.1. Fix $1 \leq p<\infty$. Assume that:

(a) $v$ is a submultiplicative weight on $\mathbb{R}^{2 d}$ such that $|v(z)| \lesssim(1+|z|)^{N_{0}}$ for some $N_{0} \in \mathbb{N}$,

(b) $m$ is a v-moderate weight on $\mathbb{R}^{2 d}$,

(c) $\psi \in C_{c}^{\infty}\left(\mathbb{R}^{2 d}\right)$.

Let $m^{*}$ be any weight function satisfying $m^{*}(N, M) \leq C_{N} m(M)$ for all $M$, $N \in \mathbb{Z}^{2 d}$. Then for every $f \in L^{2}\left(\mathbb{R}^{d}\right) \cap M_{m}^{p}\left(\mathbb{R}^{d}\right)$ we have $\psi Z f \in M_{m^{*}}^{p}\left(\mathbb{R}^{2 d}\right)$.

Proof. First we note that $\psi Z f \in L^{2}\left(\mathbb{R}^{2 d}\right)$.

Let $g \in C_{c}^{\infty}\left(\mathbb{R}^{d}\right)$ be supported on $[0,1]^{d}$ and nonzero on the interior of this cube. Define

$$
\xi=(1, \ldots, 1) \in \mathbb{R}^{d}
$$

and

$$
g_{j}(x)=g\left(x+\frac{j-1}{2 d+1} \xi\right), \quad j=1, \ldots, 2 d+1 .
$$

We have $g_{j} \in M_{v}^{1}\left(\mathbb{R}^{d}\right)$ for each $j$. Taking the support of $g_{j}$ into consideration, if $k \in \mathbb{Z}^{d}$ then

$$
Z g_{j}(x, \omega)=e^{2 \pi i k \cdot \omega} g_{j}(x-k), \quad x \in[0,1)^{d}+k-\frac{j-1}{2 d+1} \xi .
$$


The zero set of $Z g_{j}$ is

$$
\left\{(x, \omega) \in \mathbb{R}^{2 d}: x \in k-\left(\frac{j-1}{2 d+1}\right) \xi+\partial[0,1)^{d} \cap[0,1)^{d}, k \in \mathbb{Z}^{d}, \omega \in \mathbb{R}^{d}\right\},
$$

where $\partial A$ denotes the boundary of a set $A$.

Claim 1. The functions $Z g_{j}$ have no common zeros.

To see this, assume that $Z g_{j}\left(x_{0}, \omega_{0}\right)=0$ for each $j=1, \ldots, 2 d+1$. Then for each $j$ there exists a $k_{j} \in \mathbb{Z}^{d}$ such that

$$
x_{0} \in k_{j}-\left(\frac{j-1}{2 d+1}\right) \xi+\partial[0,1)^{d} \cap[0,1)^{d} .
$$

Let $\theta_{j} \in \partial[0,1)^{d} \cap[0,1)^{d}$ be such that $x_{0}=k_{j}-\left(\frac{j-1}{2 d+1}\right) \xi+\theta_{j}$. For $j \neq j^{\prime}$ we have

$$
x_{0}=k_{j}-\left(\frac{j-1}{2 d+1}\right) \xi+\theta_{j}=k_{j^{\prime}}-\left(\frac{j^{\prime}-1}{2 d+1}\right) \xi+\theta_{j^{\prime}},
$$

so $k_{j}-k_{j^{\prime}}=\left(\frac{j-j^{\prime}}{2 d+1}\right) \xi+\theta_{j^{\prime}}-\theta_{j}$. Each of $\theta_{j}$ and $\theta_{j^{\prime}}$ must have a zero component. If they both have a zero in the $m$ th coordinate, then $k_{j}^{(m)}-k_{j^{\prime}}^{(m)}=\left(\frac{j-j^{\prime}}{2 d+1}\right) \xi^{(m)}$. This is a contradiction since $k_{j}^{(m)}-k_{j^{\prime}}^{(m)}$ is integer. Hence no $\theta_{j}$ and $\theta_{j^{\prime}}$ can have a zero in the same component. However, this is impossible as there are $2 d+1$ vectors $\theta_{j}$ each with $d$ components. This proves the claim.

Consequently, Theorem 2.6 implies that the Fourier coefficients of $Z f \overline{Z g_{j}}$ belong to $\ell_{m}^{p}\left(\mathbb{Z}^{2 d}\right)$ for each $j=1, \ldots, 2 d+1$.

Now let $\phi \in C_{c}^{\infty}\left(\mathbb{R}^{2 d}\right)$ be supported in the unit square $[0,1]^{2 d}$ and strictly positive on its interior. For $j=1, \ldots, 2 d+1$ define

$$
\phi_{j}(x, \omega)=\phi\left((x, \omega)+\left(\frac{j-1}{2 d+1}\right)(\xi, \xi)\right) \quad \text { and } \quad G_{j}=\phi_{j} Z g_{j} .
$$

Since each $g_{j}$ is Schwartz-class, we have $Z g_{j} \in C^{\infty}\left(\mathbb{R}^{2 d}\right)$. Therefore, for any $N_{1} \in \mathbb{N}$ and any weight $v_{2}$ satisfying $v_{2}(z) \lesssim(1+|z|)^{N_{1}}$ we have

$$
G_{j}=\phi_{j} Z g_{j} \in C_{c}^{\infty}\left(\mathbb{R}^{2 d}\right) \subseteq M_{v_{2}}^{1}\left(\mathbb{R}^{2 d}\right) .
$$

For the remainder of this proof, let $v_{2}$ be any such weight.

Claim 2. The zero set of $Z g_{j}$ is contained in the zero set of $\phi_{j}$.

This follows from the fact that the zero set of $Z g_{j}$ is

$$
\bigcup_{k \in \mathbb{Z}^{d}}\left(\left(-\frac{j-1}{2 d+1}\right) \xi+k+\partial[0,1)^{d} \cap[0,1)^{d}\right) \times \mathbb{R}^{d}
$$

while the zero set of $\phi_{j}$ is

$$
\mathbb{R}^{2 d} \backslash\left(-\frac{j-1}{2 d+1}(\xi, \xi)+(0,1)^{2 d}\right) .
$$


Now,

$$
Z G_{j}(x, \omega, p, s)=\sum_{(m, n) \in \mathbb{Z}^{2 d}} e^{2 \pi i(p, s) \cdot(m, n)} \phi_{j}(x-m, \omega-n) Z g_{j}(x-m, \omega-n) .
$$

Since $\phi_{j}$ is compactly supported, for $(x, \omega) \in(m, n)-\left(\frac{j-1}{2 d+1}\right)(\xi, \xi)+[0,1)^{2 d}$ we have that

$$
Z G_{j}(x, \omega)=e^{2 \pi i(p, s) \cdot(m, n)} \phi_{j}(x-m, \omega-n) Z g_{j}(x-m, \omega-n) .
$$

The zero set of $Z G_{j}$ is

$$
\bigcup_{(m, n) \in \mathbb{Z}^{2 d}}\left(\left(-\frac{j-1}{2 d+1}\right)(\xi, \xi)+(m, n)+\partial[0,1)^{2 d} \cap[0,1)^{2 d}\right) \times \mathbb{R}^{2 d} .
$$

Claim 3. The functions $Z G_{j}$ have no common zeros.

To see this, suppose $(x, \omega, p, s) \in \mathbb{R}^{2 d} \times \mathbb{R}^{2 d}$ is a common zero point for the functions $Z G_{j}$. Then for each $j=1, \ldots, 2 d+1$ there exists $\left(m_{j}, n_{j}\right) \in \mathbb{Z}^{2 d}$ and $\theta_{j} \in\left(-\frac{j-1}{2 d+1}\right)(\xi, \xi)+(m, n)+\partial[0,1)^{2 d} \cap[0,1)^{2 d}$ such that $(x, \omega)=\left(m_{j}, n_{j}\right)-$ $\left(\frac{j-1}{2 d+1}\right)(\xi, \xi)+\theta_{j}$. Each $\theta_{j}$ must have a component that is zero.

If $j \neq j^{\prime}$ and the sth component of $\theta_{j}$ and $\theta_{j^{\prime}}$ is both zero then, as in the proof of Claim 1, we obtain that $\left(m_{j}, n_{j}\right)^{(s)}-\left(\frac{j-1}{2 d+1}\right)(\xi, \xi)^{(s)}=\left(m_{j^{\prime}}, n_{j^{\prime}}\right)^{(s)}-$ $\left(\frac{j^{\prime}-1}{2 d+1}\right)(\xi, \xi)^{(s)}$. This implies $\frac{j-j^{\prime}}{2 d+1}$ is an integer, which is a contradiction. Hence no $\theta_{j}$ and $\theta_{j^{\prime}}$ can have a zero in the same component, which is impossible since the number of $j$ 's is $2 d+1$ while the dimension of $\theta_{j}$ is $2 d$.

Claim 4. There exists a $v_{2}$-moderate weight $m^{*}: \mathbb{R}^{2 d} \times \mathbb{R}^{2 d} \rightarrow(0, \infty)$ such that the sequence $c_{N,-M}$ belongs to $\ell_{m}^{p}\left(\mathbb{Z}^{2 d}\right)$, where $c_{M, N}$ are the Fourier coefficients of $Z(\psi Z f) \cdot \overline{Z G_{j}}$

To see this, fix $j$ and let $K \in \mathbb{Z}^{2 d}$ and $\alpha \in \mathbb{N}$ be such that $\operatorname{supp}(\psi) \subseteq$ $K+[0, \alpha)^{2 d}$. Write $M=\left(m_{1}, m_{2}\right), N=\left(n_{1}, n_{2}\right) \in \mathbb{Z}^{2 d}$ and $X=\left(x_{1}, x_{2}\right)$, $Y=\left(y_{1}, y_{2}\right) \in \mathbb{R}^{2 d}$, etc. Recalling that $G_{j}=\phi_{j} Z g_{j}$, we use equation (2.1) to compute that the Fourier coefficient $c_{N,-M}$ of $Z(\psi Z f) \cdot \overline{Z G_{j}}$ is

$$
\begin{aligned}
c_{N,-M}= & V_{G_{j}}(\psi Z f)(M, N) \\
= & \int_{\mathbb{R}^{2 d}} \psi(X) Z f(X) \overline{G_{j}(X-M)} e^{-2 \pi i N \cdot X} d X \\
= & \int_{K+[0, \alpha)^{2 d}} \psi(X) Z f(X) \phi_{j}(X-M) \overline{Z g_{j}(X-M)} e^{-2 \pi i N \cdot X} d X \\
= & \alpha^{2 d} \int_{[0,1)^{2 d}} \psi(\alpha Y+K) Z f(\alpha Y+K) \phi_{j}(\alpha Y+K-M) \\
& \quad \times \overline{Z g_{j}(\alpha Y+K-M)} e^{-2 \pi i N \cdot(\alpha Y+K)} d Y
\end{aligned}
$$




$$
\begin{aligned}
& =\alpha^{2 d} \int_{[0,1)^{2 d}} \psi(\alpha Y+K) \phi_{j}(\alpha Y+K-M) Z f\left(\alpha y_{1}+k_{1}, \alpha y_{2}+k_{2}\right) \\
& \quad \times \overline{Z g_{j}\left(\alpha y_{1}+k_{1}-m_{1}, \alpha y_{2}+k_{2}-m_{2}\right)} e^{-2 \pi i N \cdot(\alpha Y+K)} d Y \\
& =\alpha^{2 d} \int_{[0,1)^{2 d}} \psi(\alpha Y+K) \phi_{j}(\alpha Y+K-M) Z f\left(\alpha y_{1}+k_{1}, \alpha y_{2}\right) \\
& \quad \times \overline{Z g_{j}\left(\alpha y_{1}+k_{1}-m_{1}, \alpha y_{2}\right)} e^{-2 \pi i N \cdot(\alpha Y+K)} d Y \\
& =\alpha^{2 d} \int_{[0,1)^{2 d}} \psi(\alpha Y+K) \phi_{j}(\alpha Y+K-M) e^{2 \pi i \alpha y_{2} \cdot k_{1}} Z f\left(\alpha y_{1}, \alpha y_{2}\right) \\
& \quad \times e^{-2 \pi i \alpha y_{2} \cdot\left(k_{1}-m_{1}\right)} \overline{Z g_{j}\left(\alpha y_{1}, \alpha y_{2}\right)} e^{-2 \pi i\left(n_{1}, n_{2}\right) \cdot\left(\alpha y_{1}+k_{1}, \alpha y_{2}+k_{2}\right)} d Y \\
& =\alpha^{2 d} \int_{[0,1)^{2 d}} \psi(\alpha Y+K) \phi_{j}(\alpha Y+K-M) Z f(\alpha Y) \overline{Z g_{j}(\alpha Y)} \\
& \quad \times e^{2 \pi i\left(\alpha y_{2} \cdot k_{1}-\alpha y_{2} \cdot k_{1}+\alpha y_{2} \cdot m_{1}-n_{1} \cdot \alpha y_{1}-n_{2} \cdot \alpha y_{2}\right)} d Y \\
& =\alpha^{2 d} \int_{[0,1)^{2 d}} \psi(\alpha X+K) \phi_{j}(\alpha X+K-M) Z f(\alpha X) \overline{Z g_{j}(\alpha X)} \\
& \quad \times e^{-2 \pi i\left(n_{1}, n_{2}-m_{1}\right) \cdot\left(\alpha x_{1}+k_{1}, \alpha x_{2}+k_{2}\right)} d X
\end{aligned}
$$

where we have applied the quasiperiodicity of the Zak transform and the fact that $M, N, K$ belong to $\mathbb{Z}^{2 d}$.

Note that there are only a finite number of $M$ such that $\phi_{j}(\alpha X-M+K)$ is not identically zero on $[0,1)^{2 d}$, and hence a finite number of $M$ such that $c_{N,-M}$ is nonzero. Let $B$ be the set of those $M \in \mathbb{Z}^{2 d}$ such that $c_{N,-M} \neq 0$. Let us fix an $M \in B$ and define the following functions:

$$
\begin{aligned}
\Phi^{(M)}(X) & =\psi(\alpha X+K) \phi_{j}(\alpha X+K-M) Z f(\alpha X) \overline{Z g_{j}(\alpha X)} e^{-2 \pi i m_{1} \cdot x_{2}}, \\
U^{(M)}(X) & =\psi(\alpha X+K) \phi_{j}(\alpha X+K-M) e^{-2 \pi i m_{1} \cdot x_{2}}, \\
W(X) & =Z f(\alpha X) \overline{Z g_{j}(\alpha X)} .
\end{aligned}
$$

We note some facts about these functions.

i. $\Phi^{(M)}=U^{(M)} W$.

ii. We have

$$
\begin{aligned}
c_{N,-M} & =\alpha^{2 d} \int_{[0,1)^{2 d}} \Phi^{(M)}(X) e^{-2 \pi i \alpha N \cdot X} d X \\
& =\alpha^{2 d} \widehat{\Phi^{(M)}}(\alpha N)=\alpha^{2 d}\left(\widehat{U^{(M)}} * \widehat{W}\right)(\alpha N) .
\end{aligned}
$$

iii. $\widehat{W}(\alpha N)=\int_{[0,1)^{2 d}} Z f(\alpha X) \overline{Z g_{j}(\alpha X)} e^{-2 \pi i \alpha N \cdot X} d X=\left(Z f \overline{Z g_{j}}\right)^{\wedge}(N)$. 
iv. $U^{(M)}$ is a compactly supported infinitely differentiable function whose support lies inside the unit cube $[0,1]^{2 d}$. Consequently, $\left\|\left\{\widehat{U^{M}}(\alpha N)\right\}\right\|_{\ell_{v}^{1}\left(\mathbb{Z}^{2 d}\right)}<$ $\infty$ for any polynomial weight function $v$ defined on $\mathbb{R}^{2 d}$.

v. By Theorem 2.6, the hypothesis $f \in M_{m}^{p}\left(\mathbb{R}^{2 d}\right)$ implies that the Fourier coefficients of $Z f \overline{Z g_{j}}$ belong to $\ell_{m}^{p}\left(\mathbb{Z}^{2 d}\right)$, and therefore $\{\widehat{W}(\alpha N)\} \in \ell_{m}^{p}\left(\mathbb{Z}^{2 d}\right)$.

Fix now any weight function $m^{*}: \mathbb{R}^{2 d} \times \mathbb{R}^{2 d} \rightarrow \mathbb{R}$ such that:

- there exists a $C>0$ such that $m^{*}(M, N) \leq C m(N)$ for all $N \in \mathbb{Z}^{2 d}$ and $M \in B$, and

- $m^{*}$ is $v_{2}$-moderate with respect to some submultiplicative function $v_{2}$.

For example, we can simply take $m^{*}(M, N)=m(N)$.

Define $m_{M}(N)=m^{*}(M, N)$. Using Proposition 2.4, we compute that

$$
\begin{aligned}
\left\|\left\{c_{N,-M}\right\}_{N \in \mathbb{Z}^{2 d}}\right\|_{\ell_{m_{M}}^{p}\left(\mathbb{Z}^{2 d}\right)} & =\left(\sum_{N \in \mathbb{Z}^{2 d}}\left|c_{N,-M}\right|^{p} m^{*}(M, N)^{p}\right)^{1 / p} \\
& \leq C\left(\sum_{N \in \mathbb{Z}^{2 d}}\left|c_{N,-M}\right|^{p} m(N)^{p}\right)^{1 / p} \\
& =C \alpha^{2 d}\|\{(\widehat{U(M)} * \widehat{W})(\alpha N)\}\|_{\ell_{m}^{p}\left(\mathbb{Z}^{2 d}\right)} \\
& \leq C C^{\prime} \alpha^{2 d}\|\widehat{U}(\alpha N)\|_{\ell_{v}^{1}\left(\mathbb{Z}^{2 d}\right)}\|\widehat{W}(\alpha N)\|_{l_{m}^{p}\left(\mathbb{Z}^{2 d}\right)} \\
& <\infty .
\end{aligned}
$$

From above, we obtain

$$
\begin{aligned}
\left\|\left\{c_{N,-M}\right\}_{M, N \in \mathbb{Z}^{2 d}}\right\|_{\ell_{m^{*}}^{p}\left(\mathbb{Z}^{2 d} \times \mathbb{Z}^{2 d}\right)} & =\left(\sum_{N \in \mathbb{Z}^{2 d}} \sum_{M \in B}\left|c_{N,-M}\right|^{p} m^{*}(M, N)^{p}\right)^{1 / p} \\
& =\left(\sum_{M \in B} \sum_{N \in \mathbb{Z}^{2 d}}\left|c_{N,-M}\right|^{p} m^{*}(M, N)^{p}\right)^{1 / p} \\
& \leq\left(\sum_{M \in B}\left\|\left\{c_{N,-M}\right\}_{N \in \mathbb{Z}^{2 d}}\right\|_{l_{m_{M}}^{p}\left(\mathbb{Z}^{2 d}\right)}^{p}\right)^{1 / p}<\infty,
\end{aligned}
$$

since $B$ is a finite set.

As an application of this theorem, we recover the following result, which is [10, Lemma 2.3]. 
Corollary 4.2. Assume $f \in H^{s_{1}}\left(\mathbb{R}^{d}\right)$ and $\widehat{f} \in H^{s_{2}}\left(\mathbb{R}^{d}\right)$ where $s_{1}, s_{2}>0$. Then for any smooth, compactly supported function $\psi \in C_{c}^{\infty}\left(\mathbb{R}^{2 d}\right)$ we have $\psi Z f \in$ $\mathcal{F} L_{m}^{2}\left(\mathbb{R}^{2 d}\right)$ where $m(x, y)=\left(1+|x|^{2 s_{1}}+|y|^{2 s_{2}}\right)^{1 / 2}$.

Proof. Note that $m(x, y)$ satisfies

$$
m(x, y) \leq\left(1+m_{s_{1}}(x)^{2}+m_{s_{2}}(y)^{2}\right)^{1 / 2} .
$$

Hence $f \in M_{m}^{2}\left(\mathbb{R}^{d}\right)$ by Proposition 2.2. Now let

$$
v(x, y)=\left(1+\left(|x|^{2}+|y|^{2}\right)^{1 / 2}\right)^{\max \left\{s_{1}, s_{2}\right\}} .
$$

Then $m$ is a $v$-moderate weight. By Theorem 4.1, $\psi Z f \in M_{m^{*}}^{2}\left(\mathbb{R}^{2 d}\right)$. Taking

$$
m^{*}(x, y, u, v)=m(u, v),
$$

by Proposition 2.1(b) we obtain $\psi Z f \in \mathcal{F} L_{m}^{2}\left(\mathbb{R}^{2 d}\right)$.

Another implication of Theorem 4.1 is that the Zak transform embeds certain modulation spaces into a local VMO space.

Corollary 4.3. Let $v$ be a submultiplicative weight satisfying $|v(z)| \leq(1+$ $|z|)^{N_{0}}$ for some $N_{0} \in \mathbb{N}$, and let $m$ be a v-moderate weight such that $m(x) \lesssim$ $\left(1+|x|^{2}\right)^{1 / 2}$. If $f \in L^{2}(\mathbb{R}) \cap M_{m}^{p}(\mathbb{R})$ where $1 \leq p \leq 2$, then $Z f \in \operatorname{VMO}(K)$ for any compact set $K \subset \mathbb{R}^{2}$.

\section{Application to the Balian-Low Theorem}

We will briefly indicate how the Classical BLT can be recovered from our results.

Given $g \in L^{2}(\mathbb{R})$ and constants $\alpha, \beta>0$, the associated Gabor system is

$$
\mathcal{G}(g, \alpha, \beta)=\left\{e^{2 \pi i m \beta x} g(x-n \alpha)\right\}_{m, n \in \mathbb{Z}} .
$$

We are interested in whether $\mathcal{G}(f, \alpha, \beta)$ is a frame for $L^{2}(\mathbb{R})$, which means that there exist $A, B>0$ such that

$$
A\|f\|_{2}^{2} \leq \sum_{m, n \in \mathbb{Z}}\left|\left\langle f(x), e^{2 \pi i m \beta x} g(x-n \alpha)\right\rangle\right|^{2} \leq B\|f\|_{2}^{2}, \quad f \in L^{2}(\mathbb{R}) .
$$

Frames are a generalization of orthonormal bases, and it is natural to consider under what conditions $\mathcal{G}(g, \alpha, \beta)$ will generates a frame. The classical BalianLow Theorem addresses this question for the case $\alpha \beta=1$ (the "critical density"), which by a change of variables can be further reduced to the case $\alpha=\beta=1$. Other Balian-Low-type theorems can be found in [1], [2], [3], [9], [10], [12]. 
Theorem 5.1 (Classical BLT). Given $g \in L^{2}(\mathbb{R})$, if $g \in H^{1}(\mathbb{R})$ and $\widehat{g} \in H^{1}(\mathbb{R})$ then $\mathcal{G}(g, 1,1)$ is not a frame for $L^{2}(\mathbb{R})$.

The approach to the proof is based on the Zak transform, which completely determines whether the Gabor system associated with a function $g$ is a frame at the critical density.

Proposition 5.2. Fix $g \in L^{2}(\mathbb{R})$. Then $\mathcal{G}(g, 1,1)$ is a frame for $L^{2}(\mathbb{R})$ if and only if $0<A^{1 / 2} \leq|Z f| \leq B^{1 / 2}<\infty$ a.e.

If a function belongs to the modulation space $M^{1}(\mathbb{R})$, which is a subspace of $L^{2}(\mathbb{R})$, then the associated Gabor system cannot be a frame for $L^{2}(\mathbb{R})$. The reason for this is given in the following proposition, whose proof can be found in $[11]$.

Proposition 5.3. (a) If $f \in M^{1}(\mathbb{R})$ then $Z f$ is continuous.

(b) If $f \in L^{2}(\mathbb{R})$ and $Z f$ is continuous then $Z f$ must have a zero.

Now we prove a BLT-type result. We word this theorem in terms of modulation spaces, but the strongest conclusion is obtained by taking $p=2$ and $m(x, w)=\left(1+|x|^{2}+|w|^{2}\right)^{1 / 2}$, which recovers the Classical BLT in its usual form.

Theorem 5.4. Let $v$ be a submultiplicative weight with $|v(z)| \lesssim(1+|z|)^{N_{0}}$ for some $N_{0} \in \mathbb{N}$, and let $m$ be a v-moderate weight such that $m(x, w) \gtrsim$ $\left(1+|x|^{2}+|w|^{2}\right)^{1 / 2}$ where $(x, w) \in \mathbb{R}^{2}$. If $f \in L^{2}(\mathbb{R}) \cap M_{m}^{p}(\mathbb{R})$ where $1 \leq p \leq 2$, then $\mathcal{G}(f, 1,1)$ is not a frame for $L^{2}(\mathbb{R})$.

Proof. If $\mathcal{G}(f, 1,1)$ is a frame then $Z f \in L^{\infty}\left(\mathbb{R}^{2}\right)$. Corollary 4.3 therefore implies that $Z f \in V M O(K)$ for any compact set $K$. Since $Z f$ is quasiperiodic, it follows from [10, Lemma 4.1] that ess $\inf |Z f|=0$, which is a contradiction.

\section{ACKNOWLEDGEMENT}

The second author was partially supported by NSF Grant DMS-0806532.

\section{References}

[1] J. J. Benedetto, W. Czaja, P. Gadziński, and A. M. Powell, The Balian-Low Theorem and regularity of Gabor systems, J. Geom. Anal., 13, 239-254, 2003.

[2] J. J. Benedetto, W. Czaja, and A. M. Powell, An optimal example for the Balian-Low uncertainty principle, SIAM J. Math. Anal., 38, 333-345, 2006. 
[3] J. J. Benedetto, W. Czaja, A. M. Powell, and J. Sterbenz, An endpoint $(1, \infty)$ Balian-Low theorem, Math. Res. Lett., 13, 467-474, 2006.

[4] A. Bényi and K. Okoudjou, Bilinear pseudodifferential operators on modulation spaces, J. Fourier Anal. Appl., 10, 301-313, 2004.

[5] H. Brezis and L. Nirenberg, Degree Theory and BMO. I. Compact Manifolds without Boundaries, Selecta Math. (N.S.), 1 (2), 197-263, 1995.

[6] I. Daubechies, The wavelet transform, time-frequency localization and signal analysis, IEEE Trans. Inform. Theory, 39, 961-1005, 1990.

[7] L. C. Evans, Partial Differential Equations, Graduate Studies in Mathematics, vol. 19, American Mathematical Society, Providence, RI, 1998.

[8] H. G. Feichtinger, Modulation spaces on locally compact Abelian groups, Technical Report, University of Vienna, 1983.

[9] J.-P. Gabardo and D. Han, Balian-Low phenomenon for subspace Gabor frames, J. Math. Phys., 45, 3362-3378, 2004.

[10] S. Z. Gautam, A critical-exponent Balian-Low Theorem, Math. Res. Letters, 15, 471-483, 2008.

[11] K. Gröchenig, Foundations of Time-Frequency Analysis, Birkhäuser, Boston, 2001.

[12] K. Gröchenig, D. Han, C. Heil, and G. Kutyniok, The Balian-Low Theorem for symplectic lattices in higher dimensions, Appl. Comput. Harmon. Anal., 13, 169-176, 2002.

[13] K. Gröchenig and C. Heil, Modulation spaces and pseudodifferential operators, Integral Equations Operator Theory, 34, 439-457, 1999.

[14] I. M. Gel'fand, Expansion in characteristic functions of an equation with periodic coefficients (Russian), Doklady Akad. Nauk SSSR (N.S.) 73, 11171120, 1950.

[15] A. J. E. M. Janssen, Zak transform characterization of $S_{0}$, Sampl. Theory Signal Image Process., 5, 141-162, 2006.

[16] K. A. Okoudjou, Embedding of some classical Banach spaces into modulation spaces, Proc. Amer. Math. Soc., 132, 1639-1647, 2004.

[17] D. Sarason, Functions of vanishing mean oscillation, Trans. Amer. Math. Soc., 207, 391-405, 1975. 
[18] T. Strohmer, Pseudodifferential operators and Banach algebras in mobile communications, Appl. Comput. Harmon. Anal., 20, 237-249, 2006.

[19] T. Strohmer and S. Beaver, Optimal OFDM system design for timefrequency dispersive channels, IEEE Trans. Communications, 51, 1111$1122,2003$.

[20] T. Strohmer and R. W. Heath, Jr., Grassmannian frames with applications to coding and communication, Appl. Comput. Harmon. Anal., 14, 257-275, 2003.

[21] J. Toft, Continuity properties for modulation spaces, with applications to pseudo-differential calculus, I, J. Funct. Anal., 207, 399-429, 2004.

[22] J. Toft, Convolutions and embeddings for weighted modulation spaces, in: Advances in Pseudo-Differential Operators, R. Ashino et al., eds., Birkhäuser, Basel, 2004, 165-186.

[23] D. F. Walnut, Lattice size estimates for Gabor decompositions, Monatsh. Math. 115, 245-256, 1993. 